\title{
Determination of threats traceability in the use of explosive materials in different environments
}

\author{
Gabriel Vasilescu, ${ }^{1,}$, Robert Laszlo ${ }^{1}$, Attila Kovacs $^{1}$, Ilie Ciprian Jitea ${ }^{1}$, Daniela Rus ${ }^{1}$, \\ Cristian Radeanu ${ }^{1}$, and Claudia Miron ${ }^{1}$ \\ ${ }^{1}$ National Institute for Research and Development in Mine Safety and Protection to Explosion - \\ INSEMEX, 32-34 General Vasile Milea Str. 332047, Petroşani, Hunedoara County, Romania
}

\begin{abstract}
The paper presents the results of the theoretical and practical research on the detection and examination of the threat traces taken from samples of explosive substances from experimental attempts on explosive materials in various working environments. The use of I-SCAN LSD highperformance specialized equipment that has been purchased within the project, has enabled the detection and examination of small amounts of low volatility organic substances on the basis of ion mobility spectrometry as a result of explosive testing at different locations at the level of the industrial site within the INSEMEX Polygon and quantifying the degree of contamination of contaminated objects.
\end{abstract}

\section{Introduction}

Detecting and identifying explosives are important stages of judicial or intervention action to prevent and combat both crime and the implementation of terrorist threats, a violent and destructive plague of contemporary society. Illicit use of firearms and explosives by terrorist organizations or other groups or individuals is a global issue of contemporary society. The problem becomes even more complex when it comes to researching samples taken from where an explosive incident occurred. The difficulty comes from the destruction and scattering of incident evidence from the explosion, and from the fact that these chemicals are difficult to recognize, requiring qualified and well-trained investigators to correctly select residues, assessing the location of the explosive device as accurately as possible, the most likely directions of fragments spreading resulting from detonation, etc.

Analysis of evidence to trace the threat of explosive material traces and identifying the material for forensic investigations undertaken for the detection of perpetrators are complex, laborious and difficult to accomplish. It follows that the success of such an investigation is interdependent with the quality of the harvested samples, with the perception of some forensic tactics and actions, but also with the endowment with performing analytical means, considering that in these situations the pressure under which results are required faster to start the investigation requires shorter analysis time [1-2]. If laboratory analysis techniques benefit from the static location of investigation systems, which are reflected in the accuracy of the results, those used to detect explosives on the

*Corresponding author: dragos.vasilescu@insemex.ro 
field in a very short time, must overcome the barrier of dimensional and mass limitation and obtain operative results at the highest levels of sensitivity and selectivity. Such systems must allow the detection (eventual identification) of explosive quantities at ultra-low, nano and even picogram levels, this feature being called sensitivity from a broader range of explosives, that is, having a high level of selectivity [3].

Trinitrotoluene (TNT), dinitrobenzene (DNB), picric acid, pentaerythritol tetranitrate (PETN), cyclotrimethylene trinitramine (RDX), mixtures thereof and, in special applications, cyclo tetra methylene tetranitramine (HMX) are explosive materials most commonly used in the military field. Many of the explosives contain, besides useful substances, small amounts of paraffins, oils, plasticizers, stabilizers or other technological additives, some of which also have the role of "marker" of the substance / mixture.

In the industrial and commercial fields, the explosives used are based on ammonium nitrate, nitrocellulose and / or nitro-glycerine and mixtures thereof. Extruded crafts (improperly known as improvised explosives) may be of the above-mentioned categories or may be peroxidic in nature (TATP, HMTD s.o.).

What characterizes the explosives with few exceptions, is the presence of nitrogen atoms in their molecules. Organic materials also contain $\mathrm{C}, \mathrm{H}, \mathrm{N}$ atoms, while inorganic materials don't contain $\mathrm{C}$, but only $\mathrm{H}$ and $\mathrm{N}$. In view of the above, it is found that in most cases the explosives have the sum of the $\mathrm{C}$ and $\mathrm{H}$ atoms higher than $\mathrm{O}$ and $\mathrm{N}$, having a characteristic $\mathrm{O} / \mathrm{N}$ ratio. The oxygen depletion (excess) contained in the molecule so that the reaction results in complete combustion products only, so that the thermal effect of the reaction is maximum, is expressed by the "oxygen balance".

Also, explosive materials - whether solid, but especially liquid - release vapours (a phenomenon enhanced by the increase in temperature) and a gaseous phase with a certain pressure, known as vapor pressure, is accumulated at their surface. Vapor pressure is often expressed as a relative concentration in the air and less often as a pressure.

Thus, the following expressions are now practiced: parts / million (ppm): 1: 106 - one molecule per million molecules of air; parts / billion (ppb): 1: 109 - one molecule per billion molecules of air; parts / trillion (ppt): 1: 1012 - one molecule per trillion of air molecules.

This feature of explosives is a valuable indicator in their detection and becomes less important in the detection of explosive mixtures where vapor pressure values are much lower than separate components (even when they are present in the same amount), because they are embedded in matrixes type: polymers, oils, resins or grease, as well as their "entrapment" in different types of packaging, the detection may also be influenced by weather or environmental conditions, depending on the technology used.

The acts of terrorism committed throughout the world, many of which have catastrophic consequences, have called into question the need to prevent attacks involving, in most cases, explosive substances. For this, detection of explosives at the highest level of performance is a decisive factor, even when they are "locked" into the body of an improvised explosive device, or when they are not part of the commercial circuit, being crafted.

In order to help identify explosives, manufacturers have implemented various marking solutions so that whether they are detecting the explosive charge from an improvised explosive device or identifying the substance used in an event (post analyzes -incident) can be easily done. The issue of craftsmanship is more complex and can only be solved by increasing the selectivity and sensitivity of the detection and identification technique, but also by updating the techniques and methods of detection and identification to the "trends" of the terrorist threats, namely to the new types of materials used, to the improvised explosive devices, or how to dismantle and place them. 
The "marking" of explosives has constantly gained ground, with even an institutionalized framework for its deployment - an international convention that has been in operation since 1998 - and numerous scientific forums in which solutions are known and diversified.

Markers are the substances introduced into the explosive composition in small percentages, to facilitate detection (detection marker) or product identification (marker for identification in post-incident investigations). Depending on the type of explosive, the embedding technique, the price elevation, the manufacturer and the application, there are several types of markers, some already successfully used, others in the experimentation or implementation phase, the problem of marking explosives remaining a challenge for researchers in the field.

Until now, for the established methods chemical substances, radiofrequency microchips and microparticles have been used as markers. In the first category are included chemicals that have a much higher vapor pressure than the explosive itself, such as ortho and para mononitrotoluene (o-MNT, p-MNT), dimethyldinitrobutane (DMDNB or DMNB). Ethylene glycol dinitrate (EGDN) has volatility close to that of NG, but its presence makes it possible to detect explosive vapours with low vapor pressure or in the form of plastic explosives (eg dual-purpose explosives - civilian and military - such as SEMTEX). Radio frequency microchips can be detected with radio monitoring systems, currently only used for pharmaceutical and cosmetic labelling.

\section{Detection and examination of explosive characteristics using high performance specialized equipment (I-SCAN LSD)}

In order to develop the technical identification and technical expertise of hazardous / toxic / forbidden substances for the assessment of the risk associated with the major accidents that can occur at the level of explosives for civilian use, the Department of Explosives and Pyrotechnic Articles purchased a state-of-the-art high-performance I-SCAN LDS type equipment that was designed to detect low volatile organic substances on a portable sensor for the detection of explosives, drugs and toxic substances in the air around objects examined on the surface of different objects or on fingers and clothing (Figure 1) [1, 4].

The LSD I-SCAN device is designed on the basis of ion mobility spectrometry (IMS) and can be used to examine territories, premises, movable objects and goods at checkpoints during border control, in forensic laboratories of different departments, analytical laboratories of industrial enterprises and scientific research institutions.

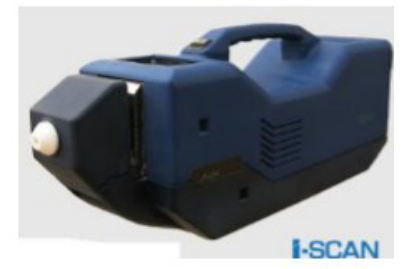

Fig. 1. Equipment for detection and examination of explosive substances, I-SCAN LSD

The LSD I-SCAN detector operating system analyses the spectral maxima of the investigated substances based on the mathematical expectation (aggregate mean) and time dispersion, corresponding to the target substance pick-ups in the system library.

I-SCAN also includes a device that allows both the sampling of air containing vapours and suspended particles and the collection of particulates collected on a special blade. 
The I-SCAN LSD specialized equipment (figure 2) applies the principles of the tested and reliable method of ion mobility spectrometry. This process is based on the separation of ions from substances, depending on mobility, while moving them into the constant electric field drift tube. Generally, vapours and particles are absorbed or remain on the surface of objects or persons they come into contact with, where the vapours can be collected and released into the chamber of the device, or the residues may be collected by wiping the surface and inserted into the apparatus, using a tester. The detector continuously sucks the air around the inspected object at a speed of between 5 and $10 \mathrm{~cm}^{3} / \mathrm{s}$. The air containing the molecules from the target substance is absorbed, and these are transmitted to the ionizing source using a corona pulse discharge where the molecules are partially ionized [4].

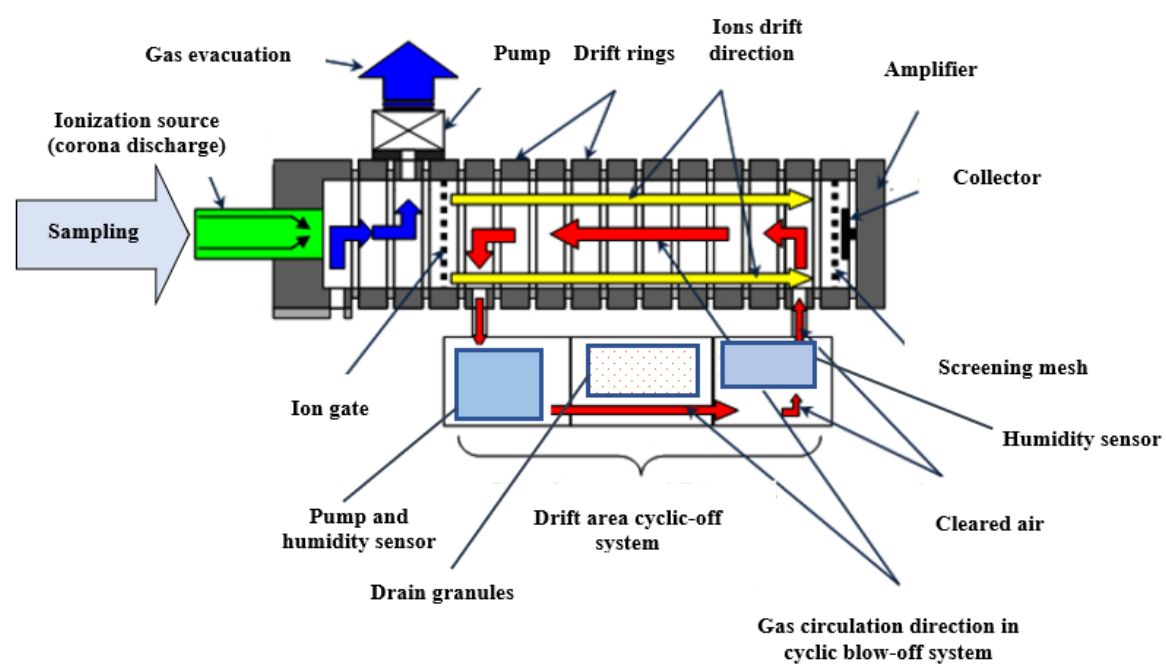

Fig. 2. Principle of operation of the I-SCAN LSD equipment scheme

The ionization process goes through several stages. When the device is active, the positive and negative ions in the ambient air (reactant ions) are aspirated into the discharge chamber (ionizing source), where their concentration is analysed. When the target substances enter the device, the reactants transfer a charge to the related molecules based on the chemical ionization mechanism at atmospheric pressure. The non-ionized molecules of the target substances and the air are removed from the system, and the ions received are held in the ionization chamber by means of the ion barrier. After a short period of time, the ion barrier opens, and ion fractions penetrate into the drift tube with the gradient of the electric field $\mathrm{E}(\mathrm{V} / \mathrm{cm})$. The operating frequency of the ion source is about $12 \mathrm{~Hz}$, ie the system analyses 12 samples of the air collected per second.

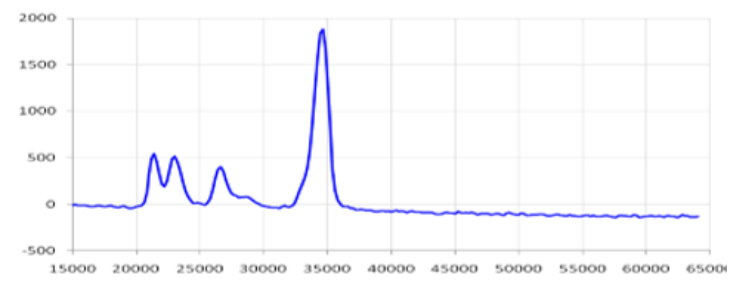

Fig. 3. Grid ion dependency curve and drift time curve 
The results are constantly compensated, and the extreme deviations caused by random fluctuations in the composition of ionic grid current and electrical disturbances are eliminated, while compensated results are equalized, results that can be presented in the form of ion mobility "spectrum" (graphic analysis). On the dependence curve of the grid ionic current expressed in conventional (ordered) units and the drift time expressed in $\mu \mathrm{s}$ (abscissa), there are peaks corresponding to ions with different mobilities (Figure 3).

The detector software analyses the received peak range, which corresponds to target substances stored in the database, based on the mathematical expectation (aggregate average) and time dispersion. If a target substance is found and the height of its peak is above the detection threshold, the detector sends an alarm signal with the marker (code) of the detected substance. I-SCAN LSD also has a dual sampling function, which allows both the removal of suspended particulate vapor and particulate matter and the collection of particulates collected on a special sampling tester

\section{Tests on detection and examination of threat trace from testing explosive materials in different work environments}

Identification of explosive substances based on the rapid detection and examination of specific characteristics was carried out on the basis of the PL-01/2017 working procedure, using the I-SCAN LSD performance equipment, designed to detect low amounts of organic substances with low volatility on the basis of ion mobility spectrometry [4-5]. The following are the results of tests in the field of detection and examination of threat traces resulting from the detonation of explosive materials in different working environments.
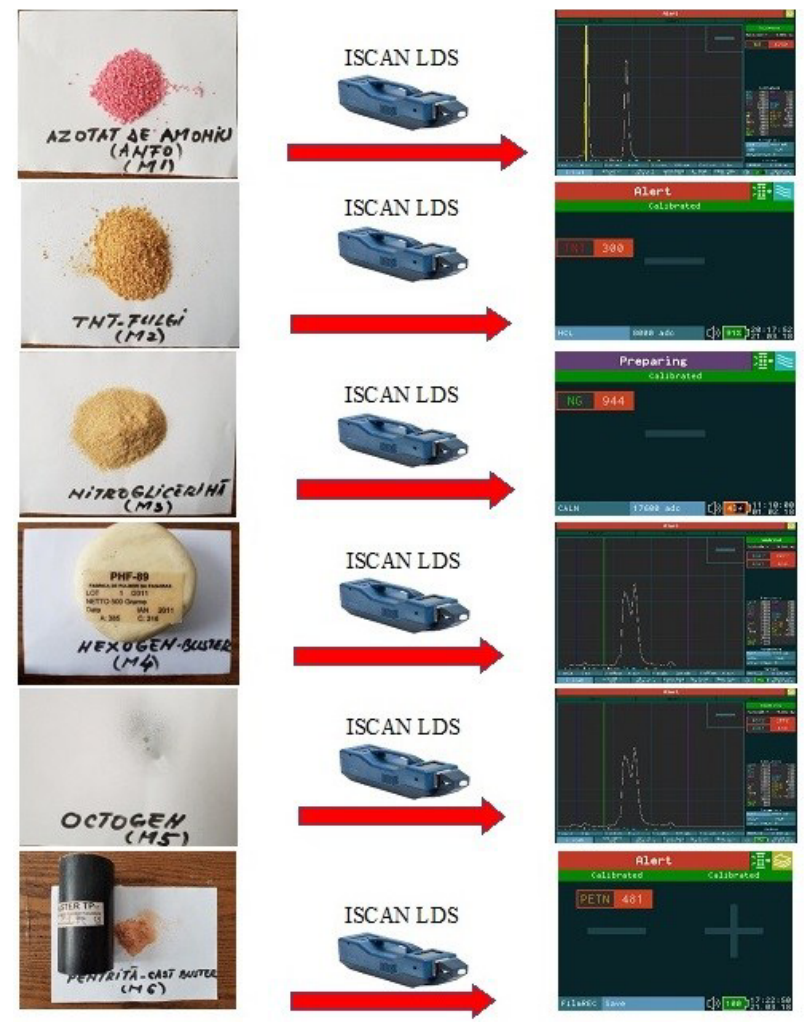

Fig. 4. Threat traces resulted from the sampling of different samples of substances 
Threat traces resulted from the sampling of different samples of substances are shown in Figure 4. Detection of threat traces resulted from the sampling of explosives collected in different working environments are shown in Figures 5-8.
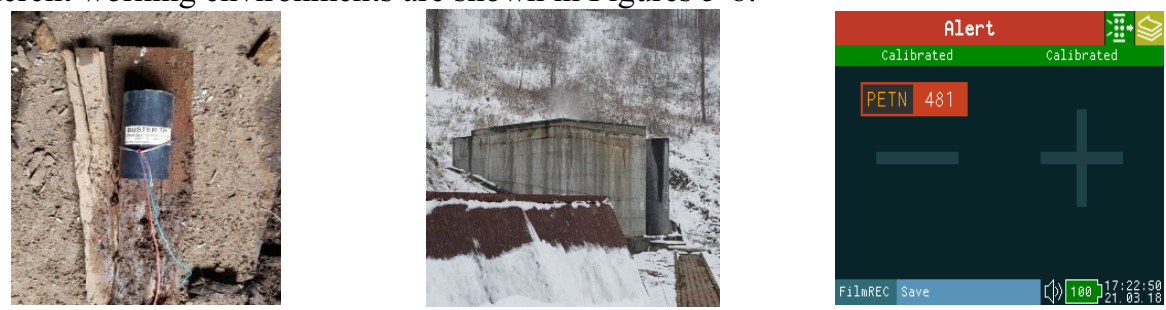

Fig. 5. Determination of threat trace to the detonation of a buster explosive charge in the explosive matter bunker within the INSEMEX Polygon
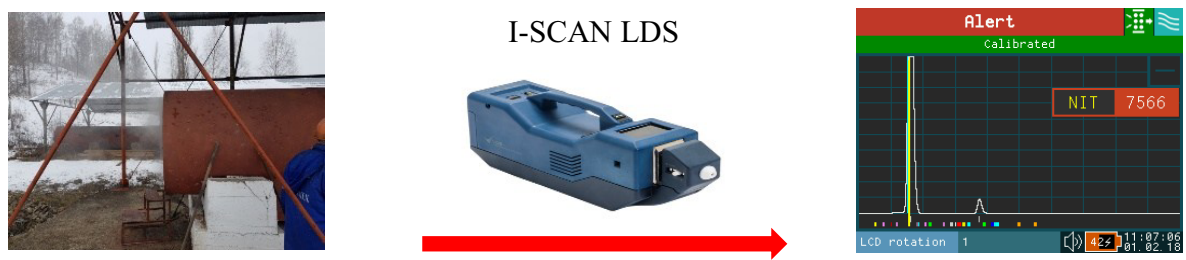

Fig. 6. Determination of threat traces of detonation of an explosive charge in the tunnel for flameproof testing.
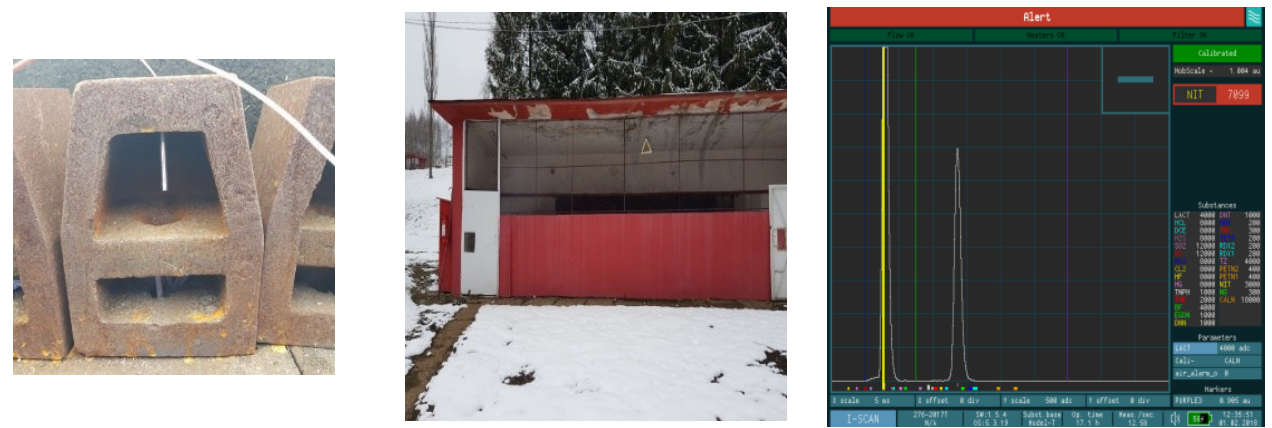

Fig. 7. Determination of the threat trace to an electric detonator in the stand for explosive initiation means. 

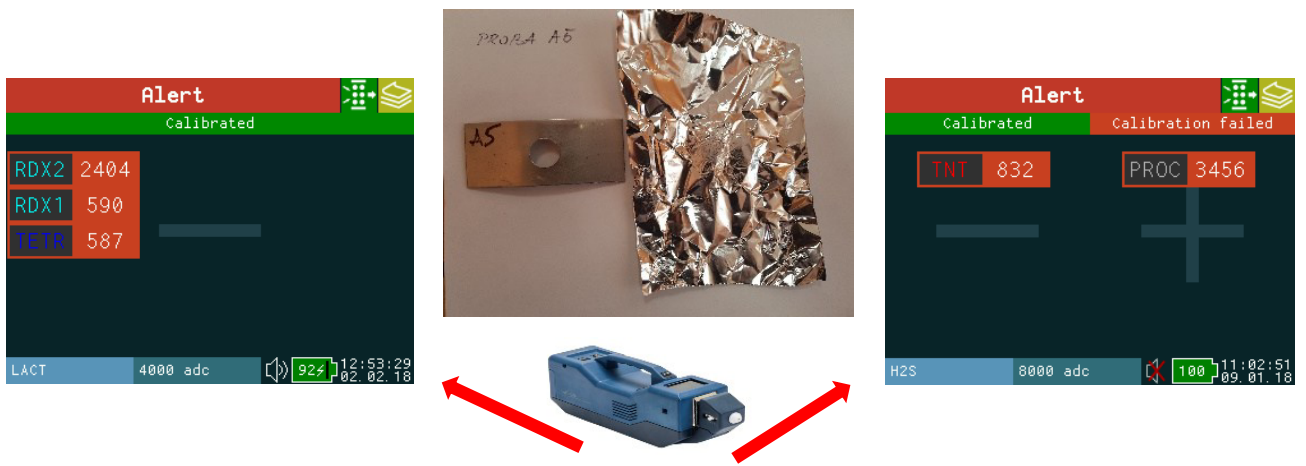

Fig. 8. Detection of threat traces from a contaminated metal plate following controlled detonation of an explosive charge in the explosive test bunker

\section{Interpretation of the results obtained}

The results of experimental research on explosive materials detonated in different working environments from the point of view of the threat traceability from analysed explosives samples or from controlled explosions conducted in the INSEMEX Polygon, highlight the presence of explosive substances detected and examined using the I-SCAN LSD specialized equipment, confirming the qualitative accuracy of the result indicators in full agreement with the nature and chemical composition of the explosive products tested according to the configuration and specificity of the test environment.

\section{Conclusions}

Analysis of samples for the traces detection of explosive materials and identification of the material for technical or criminal investigations (illicit possession / sale of explosive substances / materials, terrorist activities, etc.) are complex, laborious and difficult activities to be achieved. They require, on the one hand, rigorous and precise investigations based on the assurance of the quality of the harvested samples, with the perception of forensic tactics and procedural actions, and on the other hand the endowment with highperformance analysis equipment and apparatus that offers the possibility to obtain real results in the shortest time. The LSD I-SCAN detector operating system analyses the spectral maxima of the investigated substances based on the mathematical expectation (aggregate mean) and time dispersion, corresponding to the target substance taken from the system library. Thus, the threat traces were determined either from samples of explosive products (ANFO, TNT flakes, NG, hexogen buster, octogen, penthrite) or from the controlled explosions that were carried out at the level of the INSEMEX Polygon (explosion charge anti-burst type, test tunnel, explosive charge for flameproof environment use, electrical detonator etc.) using different explosive materials (buster explosive charge, explosive charge for use in flameproof environment, detonating electric cap etc.).

For the implementation of the explosive detection and screening system, the PL-01 procedure for the identification of explosive substances was developed at INSEMEX Polygon, based on the rapid detection and examination of specific characteristics using high-performance specialized equipment. 
This paper was developed within the Nucleu-Programme, carried out with the support of Romanian Ministry of Research and Innovation, project no. PN-19-21-02-01, project title: Research on increasing the security degree of the technical infrastructure for the storage of explosives for civil use (in Romanian: Cercetari privind cresterea gradului de Securitate la infrastructurile tehnice destinate depozitarii explozivilor de uz civil).

\section{References}

1. Directive 2014/28/EU (2014)

2. G. Vasilescu, A. Kovacs, D. Rus, R. Laszlo, O. Miclea, Proc. of the 18th Int. Multidisciplinary Scientific Geoconference SGEM 2018 18, 625-632 (2018)

3. R.I. Moraru, G.B. Babut, L.I. Cioca, Environ. Eng. Manag. J. 13, 6, 1371-1376 (2014)

4. G. Vasilescu, Researches on increasing the security degree of the technical infrastructure for the storage of explosives for civil use (INSEMEX Research Study, Petroșani, 2016)

5. L. Ivascu, L.I. Cioca, Proceedings of the 2nd International Conference on Psychology, Management and Social Science 49, 77-80 (2014) 